\title{
Utilisation d'un isolat de protéines de pomme de terre chez le vison en croissance
}

\author{
Geneviève CHARLET-LERY, Marie-Thérèse MOREL et D. ALLAIN * \\ I.N.R.A., Laboratoire de Physiologie de la Nutrition \\ * Laboratoire des Pelages, Toisons et Fourrures \\ Centre de Recherches Zootechniques, 78350 Jouy-en-Josas
}

\begin{abstract}
Résumé
L'isolat de protéines de pomme de terre extrait par thermocoagulation des eaux rouges de féculerie selon le procédé AVEBE ou Protéine PF a été introduit au taux de 8 p. 100 dans un aliment granulé pour vison en croissance en remplacement équiazoté de farines de viande et de poisson. Il apportait 17 p. 100 des protéines totales de la ration. La présence de Protéine PF provoque un léger abaissement des coefficients de digestibilité apparente de l'énergie $(82$ à 78 p. 100) et de l'azote $(79$ à 73 p. 100) mais celui-ci est compensé par une augmentation du niveau d'ingestion alimentaire. De ce fait, la croissance des animaux est identique dans les 2 groupes $(n=24)$ de mi-juillet à fin novembre. La qualité des fourrures n'est pas modifiée.

Cet aliment, d'origine végétale, riche en protéines ( 85 p. 100 M.S.), très bien équilibré en acides aminés, pauvre en matières minérales (3,5 p. 100 M.S.) et apportant peu d'extractif non azoté ( 8 à 12 p. 100) convient bien à l'alimentation du vison en croissance, du moins pour le pourcentage auquel il a été introduit dans cet essai.
\end{abstract}

Mots clés : vison, croissance, protéines de pomme de terre.

\section{Introduction}

Les rations des visons sont le plus fréquemment présentées sous forme de pâtée humide constituée en grande partie de produits carnés à l'état frais. Cette technique est contraignante pour l'éleveur tant pour l'obtention de ces produits que pour leur stockage. Aussi de nombreuses recherches se sont-elles orientées vers l'étude des produits déshydratés riches en protéines, tant pour les mélanger aux rations humides que pour les inclure dans des formules complètes présentées en granulés.

Les farines animales sont utilisées avec succès, mais elles sont coûteuses et beaucoup d'entre elles ont une teneur en matières minérales très élevée. Depuis plusieurs années, des chercheurs ont tenté d'introduire des protéines extraites de végétaux dans les mélanges destinés aux visons. En 1977, Brown \& Evans remplacent partiellement les farines de viande et de poisson par un mélange d'extrait 
de luzerne et de tourteau d'arachide; la croissance des animaux est toutefois diminuée de 10 p. 100. En 1980, Tauson \& ALDEN mettent en évidence l'intérêt du Protamyl PF (1), protéines de pomme de terre extraites des eaux rouges de féculerie par le procédé AVEBE ; dans un mélange complexe humide, ils remplacent la moitié ou la totalité des protéines de la farine de poisson par une quantité équivalente de ces protéines, soit 22 ou 42 p. 100 des protéines totales. L'insuffisance du taux d'humidité des mélanges obtenus limite un peu l'ingestion d'aliment par les animaux et ralentit légèrement leur croissance $(5 \mathrm{p}$. 100 pour les mâles, 7 p. 100 pour les femelles), mais la qualité des peaux, jugée d'après les prix de vente, n'est pas modifiée. Ces auteurs conseillent donc de limiter le pourcentage d'introduction des protéines de ce produit par rapport aux protéines totales à $17 \mathrm{p} .100 \mathrm{en}$ phase croissance-fourrure et à 5 p. 100 durant la lactation. Ils recommandent, de plus, de n'utiliser que les protéines PF à faible taux de solanine par suite de la toxicité de celle-ci.

SANDo Lund introduit 40 p. 100 de protéines de pomme de terre dans un aliment vison et n'observe aucune différence dans la taille et la qualité des peaux obtenues.

L'utilisation des Protéines PF a été étudiée chez les jeunes d'autres espèces animales. Leur apport maximum serait de 5 p. $100 \mathrm{chez}$ des poulets âgés de moins de 6 semaines (Helder \& Versteegh, 1977). Chez le porcelet précocement sevré ou non, la croissance n'est pas modifiée par un apport de 3 à 6 p. 100 (LiNDER et al., 1981) ou même de 16 p. 100 (SEve, 1977) ; inversement, BeKaERT \& EEcKhou' (1980) observent une moins bonne croissance et un moins bon indice de consommation lors d'une introduction de 3 à 4 p. 100. Ce résultat contredit les observations. effectuées par Eeckнout (1980), où la «Lysamine » apparaît comme l'une des meilleures protéines végétales pour des porcs en croissance-finition, très proche et parfois supérieure à la poudre de lait écrémé (coefficients de digestibilité et bilan N.). NOORDEWIER \& VON KEMPEN (1983 a) vont jusqu'à remplacer totalement la poudre de lait écrémé par du Protamyl dans des aliments veaux à partir de $80 \mathrm{~kg}$. Ce n'est que pour les apports les plus élevés qu'ils observent un ralentissement de croissance et l'abaissement des coefficients de digestibilité des protéines et des lipides.

La composition chimique de cet isolat de protéines, séparé des « eaux de fruits » de pomme de terre de féculerie par thermocoagulation, rend particulièrement intéressante son introduction dans les mélanges alimentaires destinés aux visons. Très riche en matières azotées $(85,2$ p. 100 M.S.) et pauvre en matières minérales $(2,85$ p. 100 M.S.), données comparables à celles obtenues par les chercheurs déjà cités (tabl. 1), il apporte peu de glucides que l'on sait mal digérés par les visons (Simoes Nunes et al., 1984). De plus l'équilibre de ces acides aminés est comparable à celui de la farine de poissons (tabl. 2), équilibre déjà mis en évidence par EeckHout (1980).

Nous avons donc décidé d'étudier les conséquences de l'introduction de la Protéine PF dans les aliments présentés sous forme de granulé chez le vison en croissance. Utilisée chez le vison totalement sevré, la présentation en granulé conduit à des croissances identiques à la présentation en pâtée humide (RougEOT et al., 1971).

(1) Selon les auteurs et les pays où sont effectuées les recherches, les protéines de pomme de terre extraites par thermocoagulation sont dénommées Protamyl PF, Protéine PF, Lysamine, Protéines de pomme de terre. 
TABLEAU 1

Composition chimique de la Protéine PF (p. 100 M.S.).

Chemical composition of potato Protein (p. 100 D.M.).

\begin{tabular}{|c|c|c|c|c|}
\hline Références & $\begin{array}{l}\text { Charlet-LeRY } \\
\text { et al. }\end{array}$ & $\begin{array}{l}\text { EECKHOUT } \\
(1980)\end{array}$ & $\begin{array}{l}\text { SEVE } \\
(1977)\end{array}$ & $\begin{array}{c}\text { LINDNER } \text { et al. } \\
\text { (1982) }\end{array}$ \\
\hline $\begin{array}{l}\text { Matière sèche } \ldots \ldots \cdots \cdots \\
\text { Dry matter }\end{array}$ & 90,4 & 91,6 & 93,0 & 90,2 \\
\hline $\begin{array}{l}\text { Protéines }(\mathrm{N} \times 6,25) \ldots \\
\text { Crude protein }\end{array}$ & 85,2 & 82,5 & 81,2 & 85,0 \\
\hline $\begin{array}{l}\text { Matières grasses } \ldots \ldots \ldots \\
\text { Fat }\end{array}$ & - & $\begin{array}{l}1,6(4,1) \\
(1)\end{array}$ & 3,5 & 3,1 \\
\hline $\begin{array}{l}\text { Cellulose brute .......... } \\
\text { Crude fibre }\end{array}$ & - & - & 0,5 & 0 \\
\hline $\begin{array}{l}\text { Matières minérales } . . . \\
\text { Mineral matter }\end{array}$ & 2,9 & 3,3 & 3,0 & 3,6 \\
\hline $\begin{array}{l}\text { Extractif non azoté (2) .. } \\
N \text { free extract }\end{array}$ & - & $\begin{array}{l}12,6(10,1) \\
(1)\end{array}$ & 11,8 & 8,4 \\
\hline
\end{tabular}

(1) Selon méthode d'analyse - According to analytical method.

(2) Calculé par différence - Calculated by difference.

TABLeau 2

Composition en acides aminés de la Protéine PF et d'une farine de poisson (1). Amino acid composition of potato Protein and fish meal ( $\mathrm{g} \%$ product).

\begin{tabular}{|c|c|c|}
\hline & $\begin{array}{c}\text { Protéine PF } \\
\text { Potato Protein }\end{array}$ & $\begin{array}{l}\text { Farine de poisson } \\
\text { Fish meal }\end{array}$ \\
\hline$\ldots \ldots \ldots \ldots \ldots \ldots \ldots \ldots$ & 93 & 92 \\
\hline Dry matter & & \\
\hline Matières azotées totales $\ldots \ldots \ldots \ldots \ldots \ldots$ & 76,5 & 71,6 \\
\hline Total crude protein & & \\
\hline Lysine . . . . . . . . . . . . . . . . & 5,40 & 5,48 \\
\hline 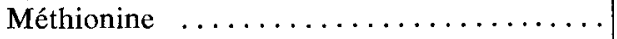 & 1,50 & 2,08 \\
\hline Méthionine + cystine $\ldots \ldots \ldots \ldots \ldots$ & 2,79 & 2,80 \\
\hline Tryptophane $\ldots \ldots \ldots \ldots \ldots \ldots \ldots$ & 0,92 & 0,79 \\
\hline Thréonine $\ldots \ldots \ldots \ldots \ldots \ldots \ldots$ & 3,86 & 3,06 \\
\hline Glycine + sérine $\ldots \ldots \ldots \ldots \ldots \ldots$ & - & 7,12 \\
\hline Leucine $\ldots \ldots \ldots \ldots \ldots \ldots \ldots$ & 6,88 & 5,20 \\
\hline Isoleucine $\ldots \ldots \ldots \ldots \ldots \ldots \ldots \ldots$ & 3,98 & 3,42 \\
\hline 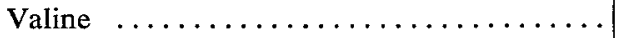 & 4,82 & 3,88 \\
\hline Histidine $\ldots \ldots \ldots \ldots \ldots \ldots \ldots \ldots$ & 1,53 & 1,61 \\
\hline Arginine $\ldots \ldots \ldots \ldots \ldots \ldots \ldots \ldots$ & 3,98 & 3,92 \\
\hline Phénylalanine + tyrosine $\ldots \ldots \ldots \ldots$ & 7,80 & 4,85 \\
\hline
\end{tabular}

(1) L'alimentation des animaux monogastriques : porc, lapin, volaille, 1984. Ed. I.N.R.A. 


\section{Conditions expérimentales}

Deux régimes isoprotéiques ont été constitués; l'un contient les farines animales habituellement utilisées (poisson, viande) partiellement remplacées dans l'autre régime par 8 p. 100 de Protéine PF, apport qui représente 17 p. 100 des protéines du régime.

La composition centésimale et l'analyse chimique des mélanges sont indiquées dans le tableau 3. Les granulés $(\varnothing: 2,5 \mathrm{~mm})$, placés dans des trémies, et l'eau sont offerts ad libitum. Pour éviter le gaspillage, les animaux n'ont accès à la trémie que par un trou rond dont le diamètre est à peine supérieur à la largeur de leur tête.

A la mi-juillet, 48 mâles Pastel âgés de 10 semaines et pesant environ $1 \mathrm{~kg}$ sont choisis parmi les animaux de la visonnerie de l'I.N.R.A. pour constituer 2 lots homogènes (tabl. 4).

\section{Tableau 3}

Composition des mélanges alimentaires et données analytiques.

Composition of food mixtures and chemical analysis.

\begin{tabular}{|c|c|c|}
\hline Ingrédients & & \\
\hline 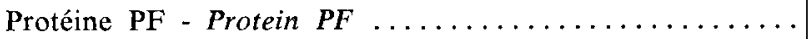 & 0 & 8 \\
\hline Farine de poisson (70 MAT) - Fish meal (70 CP) . . . . . & 20 & 16 \\
\hline Farine de viande $(80 \mathrm{MAT})-$ Meat meal $(80 \mathrm{CP}) \ldots \ldots \ldots$ & 18 & 13 \\
\hline 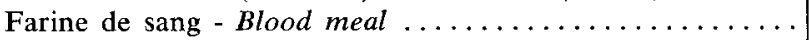 & 5 & 5 \\
\hline 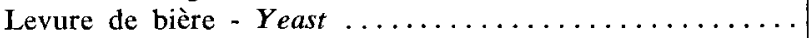 & 5 & 5 \\
\hline Pulpe de betterave - Beet pulp $\ldots \ldots \ldots \ldots \ldots \ldots$ & 2 & 2 \\
\hline 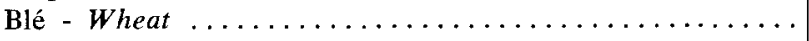 & 13 & 13 \\
\hline 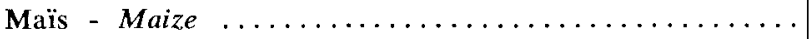 & 12,6 & 12,6 \\
\hline Germe de blé - Wheat germ $\ldots \ldots \ldots \ldots \ldots \ldots \ldots \ldots \ldots \ldots \ldots \ldots$ & 4 & 4 \\
\hline Suif $\ldots$ Tallow . . . . . . . . . . . & 16 & 17 \\
\hline Huile de tournesol - Sunflower oil . . . . . . & 2 & 2 \\
\hline Complément minéral (1) - Mineral mixture $\ldots \ldots \ldots \ldots$ & 1,6 & 1,6 \\
\hline Complément vitaminique $(2)-$ Vitamin mixture $\ldots \ldots \ldots$ & 0,8 & 0,8 \\
\hline
\end{tabular}

Composition analytique p. 100 M.S. (analytical results \% DM)

\begin{tabular}{|c|c|c|}
\hline Matière sèche - Dry matter ....... & 89,35 & 92,21 \\
\hline Matière minérale - Mineral matter & 9,34 & 9,47 \\
\hline Matières azotées - Crude protein ... & 41,34 & 38,90 \\
\hline $\mathrm{N}$ (Protéine $\mathrm{PF}$ ) / p. $100 \mathrm{~N}$ total ... & $\longrightarrow$ & 17,0 \\
\hline EB Kcal/kg MS - Energy Kcal/kg DM & 5627 & 5574 \\
\hline EM Kcal $/ \mathrm{kg}$ MS - ME Kcal/kg DM & 4315 & 4023 \\
\hline
\end{tabular}

Ajouté en dilution pour $100 \mathrm{~kg}$ d'aliment (added in $100 \mathrm{~kg}$ feed) : $125 \mathrm{~g}$ terraflavine 80 et $1 \mathrm{~g}$ $\mathrm{BHT} / \mathrm{kg}$ graisse ajoutée.

(1) et (2) In Charlet-Lery et al., 1984. 


\section{TABLEAU 4}

Poids, gain de poids et ingéré.

Live weight, weight gain and ingested food.

\begin{tabular}{|c|c|c|c|}
\hline \multirow[b]{2}{*}{ Protéine P.F. . . . . . . } & \multicolumn{3}{|c|}{$\mathrm{g} \pm \mathrm{Sm}$} \\
\hline & 0 & 8 & \\
\hline Poids initial $\ldots \ldots \ldots \ldots$ & 1026,4 & 1023,3 & N.S. \\
\hline Initial body weight & $\pm \quad 24,3$ & $\pm \quad 22,8$ & \\
\hline Gain de poids $(127 \mathrm{j})$. & 982,8 & 1032,4 & N.S. \\
\hline Weight gain $(127$ d) & $\pm \quad 49,4$ & $\pm \quad 39,0$ & \\
\hline Matière sèche ingérée $/ j \ldots$ & 86,53 & 91,83 & $0,05<\mathrm{P}<0,10$ \\
\hline Ingested dry matter/d & 2,40 & 1,54 & \\
\hline
\end{tabular}

Les quantités d'aliment ingéré sont contrôlées individuellement toutes les semaines; les animaux sont pesés toutes les 2 semaines.

A la fin d'octobre, 4 sujets sont choisis dans chacun des lots et placés dans des cages à bilan nutritionnel (Charlet-Lery et al., 1984). Deux périodes de 4 jours sont étudiées : ingesta et excréta (fèces et urine) sont journellement mesurés et échantillonnés puis analysés afin d'établir les coefficients de digestibilité de la matière sèche, de l'énergic et de l'azote et le rendement en énergie métabolisable (EM).

La longueur des peaux a été mesurée au $\mathrm{cm}$ près entre la pointe du museau et la base de la queue. Comme de coutume, ce sont des experts professionnels qui ont jugé, par comparaison, de la qualité de ces peaux.

\section{Résultats}

\section{A. Croissance et consommation}

Le tableau 4 indique le poids initial de chacun des deux lots et la croissance moyenne des animaux durant les 127 jours d'expérience : la légère différence en faveur des animaux recevant la Protéine PF n'est pas significative. Des gains de poids pratiquement identiques sont obtenus avec une légère augmentation $(+6 \mathrm{p} .100)$ de la matière sèche ingérée par le lot Protéine PF, comparativement au lot témoin : $86,5 \pm 2,4 \mathrm{~g}$ contre $91,8 \pm 1,5 \mathrm{~g}$ (tabl. 4 ).

\section{B. Digestibilité et énergie métabolisable}

Lors du bilan nutritionnel, comme pendant toute l'expérience "croissance ", les animaux du lot Protéine PF ont consommé plus que ceux du lot témoin, $68,7 \pm 3,7$ contre $55,1 \pm 4,0 \mathrm{~g} / \mathrm{j}$; cette différence est significative (tabl. 5). 


\section{Tableau 5}

Coefficients de digestibilité

Digestibility coefficients.

\begin{tabular}{|c|c|c|c|}
\hline \multirow{2}{*}{ Protéine FP p. 100} & \multicolumn{2}{|c|}{$\overline{\mathrm{x}} \pm \mathrm{Sm}$} & \\
\hline & 0 & 8 & \multirow[b]{2}{*}{$0,5<\mathrm{P}<1,0$} \\
\hline $\begin{array}{l}\text { Matière sèche ingérée/animal } / \mathrm{j} \\
\text { Dry matter intake/animal/d }\end{array}$ & 55,1 & 68,7 & \\
\hline
\end{tabular}

Coefficients de digestibilité (Digestibility coefficients) :

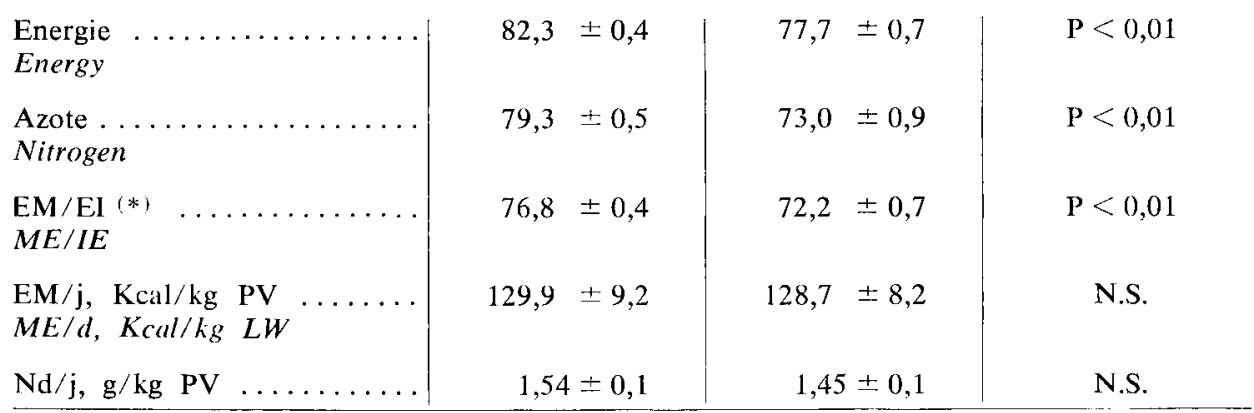

$\mathrm{Sm}:$ Ecart-type de la moyenne - Standard deviation.

(*) EI : énergie brute ingérée.

La présence de Protéine PF dans le régime abaisse significativement les coefficients de digestibilité de l'énergie (de $82,3 \pm 0,4$ à $77,7 \pm 0,7$ ), de l'azote (de $79,3 \pm 0,5$ à $73,0 \pm 0,9$ ) et le rapport $\mathrm{EM} / \mathrm{EI}$ (de $76,8 \pm 0,4$ à $72,2 \pm 0,7$ ), ce qui représente des diminutions de 5,7 p. $100,7,9$ p. 100 et 6,0 p. 100 respectivement poul: chacune des 3 données.

Mais les quantités consommées ayant été plus élevées lorsque la ration inclut la Protéine PF, les animaux soumis à ce régime ont eu à leur disposition, proportionnellement à leur poids et comparativement aux visons témoins, les mêmes quantités d'énergie métabolisable : $128,7 \pm 8,2$ et $126,9 \pm 9,2 \mathrm{Kcal} / \mathrm{j} / \mathrm{kg}$ et les mêmes apports d'azote digestible : $1,45 \pm 0,09$ et $1,54 \pm 0,11 \mathrm{~g} / \mathrm{j} / \mathrm{kg}$.

Cette compensation effectuée par les animaux qui avaient lcur aliment disponible à volonté explique qu'ils aient eu des croissances pratiquement identiques.

Si l'on utilise comme valeur énergétique des matières azotées digestibles le coefficient 4,5, l'énergie apportée par celles-ci représente respectivement 34 et 32 p. 100 de l'énergie métabolisable des régimes sans et avec Protéine PF, apport satisfaisant selon les données de GLEM-HANSEN (1980).

Les peaux étaient de même longueur dans les 2 lots et aucune différence n'a été observée en ce qui concerne la qualité de la fourrure. 


\section{Discussion}

Nos résultats montrent la plus faible valeur des coefficients de digestibilité, surtout celui des protéines, et le moindre rendement EM/EI de la ration lorsque les farines de viande et de poisson ont été partiellement remplacées par la Protéine PF. L'augmentation du niveau d'ingestion pourrait être, comme chez les autres espèces animales, une réponse soit à la dilution énergétique du régime soit à sa moindre concentration en protéines digestibles; l'égalité des gains pondéraux s'explique par les consommations identiques en énergie et en protéines digestibles. L'abaissement des coefficients de digestibilité n'est pas observé par TAuson \& Alden (1980) qui distribuent leur ration sous forme de pâtée.

Les observations effectuées durant la seconde partie de la croissance des animaux entre 1 et $2 \mathrm{~kg}$ de poids plaident donc en faveur de cet isolat. Mais les expériences effectuées par les auteurs déjà cités, sur jeunes animaux (poulets, porcelets, veaux) conduisent à des résultats contradictoires. Leur irrégularité pourrait s'expliquer par des différences dues :

— à l'animal : espèce et âge ;

- à l'importance des apponts : de 12 à 70 p. 100 des protéines de la ration, mais la proportionnalité entre quantité et réponse n'est pas rigoureuse ;

- aux différences de composition dues sans doute aux modalités d'application d'un même principe technologique : de 8 à 12 p. 100 d'extractif non azoté (sans doute du glucose et du maltose) (tabl. 1).

La solanine, glucoalcaloïde présent dans la pomme de terre, surtout si celle-ci a verdi, ne devrait pas être en cause puisqu'elle est éliminée en grande partie en cours de fabrication $(500 \mathrm{ppm}$ dans le produit raffiné contre $2000 \mathrm{ppm}$ dans le produit brut). Les études de Noordewier \& van Kempen (1983 b) montrent que la solanine ne semblerait pas être seule à l'origine des difficultés rencontrées (diarrhée, apathie, chute de poids, affaiblissement de la défense contre les maladies). En effet, ces auteurs n'observent pas, chez le veau, d'action négative de ce glucoalcaloïde, même à très forte concentration lorsque l'apport protéique de la ration est la poudre de lait écrémé. La solanine en elle-même n'étant pas la cause directe des moins bons résultats obtenus, ceux-ci seraient donc imputables à la présence simultanée de la solanine et des protéines de la pomme de terre. Ainsi, serait-on amené à limiter l'introduction de la Protéine PF dans les régimes.

\section{v. Conclusions}

L'introduction de 8 p. 100 de Protéine PF dans un aliment granulé pour vison en croissance, quantité qui apporte 17 p. 100 des protéines de la ration et économise d'autant les protéines fournies par les farines de poisson et de viande, permet des croissances identiques pour les 2 lots d'animaux; le léger abaissement des coefficients de digestibilité de l'énergie et de l'azote et du rapport EM/EI est compensé 
par une augmentation de l'ingéré sec. Il est donc parfaitement possible d'introduire la Protéine PF dans les granulés pour vison en croissance à un taux de 8 p. 100 sans aucun préjudice pour la taille et la qualité des peaux.

Reçu en janvier 1985.

Accepté en mars 1985.

\section{Summary \\ Utilization of potato protein in growing mink diets}

Using the AVEBE process, potato proteins were extracted by thermocoagulation of waste water from potato starch manufactures and included into a pelleted food mixture for growing minks. Eight percent of the product «Protein PF 》 supplied 17 p. 100 of the total dietary proteins (tabl. 3).

Two groups of 24 growing male minks were used in the experiment and 4 animals of each group were used in 2 digestibility trials.

«Protein PF » significantly reduced $(p<0.01)$ digestibility coefficients of energy (82 to 78 p. 100) and protein (79 to 73 p. 100) (tabl. 5), but as the level of feed intake was higher in the «Protein $\mathrm{PF}$ » diet $(+6 \mathrm{p}$. 100) the growth of the animals was the same in both groups from mid July till slaughter (tabl. 4). Fur quality was not altered in either group.

This protein rich plant diet (85 p. 100 DM) with an amino acid pattern comparable to that of fish meal (tabl. 2), poor in minerals (3.5 p. $100 \mathrm{DM}$ ) and supplying few carbohydrates (8-12 p. $100 \mathrm{DM}$ ) is suitable for growing mink feeding at least at the level tested in this experiment.

Key words : mink, growth, potato protein.

\section{Références bibliographiques}

Bekaert H., Eeckout W., 1980. Nouvelles sources de protéines pour porcelets. 2. - L'utilité pratique d'un concentré de protéines bactériennes (Pruteen), de protéines de pomme de terre (Lysamine), de farine de soja (Provamel) et de poudre de lait écrémé. Revue de l'Agriculture, 33, 293-304.

Brown R.G., Evans E.V., 1977. All vegetable rations for mink. Univ. Guelph Nutr. Conf. Feed Manut. Proc., 4, 82-83.

Charlet-Lery G., Fiszlewicz M., Morel M.T., Rougeot J., 1984. Variation au cours du cycle annuel de l'état nutritionnel du vison mâle adulte. I. - Poids vif, niveau d'ingestion, digestibilité, rétention azotée. Ann. Zootech., 33, 73-98.

Eeckhout W., 1980. Nouvelles sources de protéines pour porcelets. 1. - Evaluation nutritionnelle d'un concentré de protéines bactériennes (Pruteen), d'un concentré de protéines de levure (Toprina), de protéines de pommes de terre (Lysamine), de farine de soja (Provamel) et de poudre de lait écrémé. Revue de l'Agriculture, 33, 19-32.

Glem-Hansen N., 1980. The protein requirements of mink during the growth period. I. - Effect of protein intake on nitrogen balance. Acta Agric. Scand., 30, 336-344.

Helder J.F., Versteegh H.A.J., 1977. Utilisation des protéines de pomme de terre dans l'alimentation des poulets de chair (Neerl.). Inst. Pluimveconderzoek «Het Spelderholt $\gg$, Rapport $\mathrm{n}^{\circ} 161,1-10$, Rapport $\mathrm{n}^{\circ} 162,2-9$.

Lindner J.P., Burgstaller G., Huber A., 1982. Zum Einfluss von Kartoffeleiweiss auf die Mastleistung und Schlachtkörperqualität von Mastschweinen unter besonderer Berücksichtigung des Aminosäurengehaltes in den Rationen. Zuchtungskunde, 54, 303-312. 
Noordewier G.J., van Kempen G.J.M., 1983 a. L'utilisation des protéines de pomme de terre. I. - Remplacement total ou partiel du lait écrémé en poudre par les protéines de la pomme de terre. (Néerl.). Bedriffsontwikkeling, 14, 203-207.

NOORDEWIER G.J., VAN KEMPEN G.J.M., 1983 a. L'utilisation des protéines de pomme de terre. II. - L'effet de la solanine sur la croissance et l'indice de consommation des veaux de boucherie. (Neerl.). Bedrijfsontwikkeling, 14, 207-211.

Rougeot J., Melcion J.P., Charlet-Lery G., Delort-Laval J., 1971. Elevage du vison avec un aliment complet aggloméré. Ann. Zootech., 20, 259-262.

SAndo Lund R. In notice documentaire «Protéine PF», distribuée par les Etablissements Paul Doitteau (France).

SEVE B., 1977. Utilisation d'un concentré de protéines de pomme de terre dans l'aliment de sevrage du porcelet de 10 jours à 21 jours. Journées Rech. porcine en France, 9, 205-210, I.N.R.A., I.T.P. éd.

Simoes-Nunes C., Charlet-Lery G., Allain D., Rougeot J., 1984. Adaptation of exocrine pancreatic secretion in mink of diet composition. 3nd Intern. Scient. Congr. in fur animal production, Versailles, $\mathrm{n}^{\circ} 16$.

Tauson A.H., Alden E., 1980. Potato protein to growing mink kits. Vara Pälsdjur, 51, $176,179-182,185$. 